\title{
Progress in understanding the biology of nodular lymphocyte-predominant Hodgkin lymphoma
}

\section{David J. Straus}

Memorial Sloan Kettering Cancer Center, New York, NY, USA

E-mail: DAVID J. STRAUS - strausd@mskcc.org

doi:10.3324/haematol.2021.278785

I t has been 77 years since Hodgkin's paragranuloma, now known as nodular lymphocyte-predominant Hodgkin Clymphoma (NLPHL), ${ }^{1}$ was described as a clinicopathological entity by Jackson and Parker. ${ }^{2}$ They noted that "Hodgkin's paragranuloma bears little or no resemblance to a true tumor, either in its histologic picture or its clinical course." They suggested that it may be an infectious rather than a neoplastic process. They noted that it may be a precursor of the more aggressive Hodgkin's granuloma, but that "patients with Hodgkin's paragranuloma may live unembarrassed by their disease - if the pathologic picture does not change - for many years. One patient (L.C. S361256) is living and active thirty-eight years after the first proved lymph-node involvement, although the condition as shown by multiple biopsies, is still present." The observation that this entity usually has an indolent course regardless of treatment approach, and that a subset of patients does well with active surveillance, has been recently reaffirmed. ${ }^{3}$

NLPHL is rare and accounts for about $6 \%$ of Hodgkin lymphomas. ${ }^{4}$ In approximately $1 \%$ of patients per year, the disease transforms into diffuse large B-cell lymphoma (DLBCL), which is associated with a shorter survival and is the major indication for treatment. 3,5 The tumor lymphocyte-predominant (LP) cells, initially termed L\&H cells ${ }^{6}$ (for their morphological resemblance to lymphocytes and histiocytes), are morphologically distinct from the ReedSternberg cells of classical Hodgkin lymphoma, having multilobulated nuclei, indistinct nucleoli, and a germinal center B-cell phenotype and genotype. ${ }^{6,7}$

In this issue of Haematologica, Paschold and colleagues describe their research into the molecular determinants of transformation of NLPHL to DLBCL. ${ }^{8}$ Immunoglobulin heavy chain next-generation sequencing and $\operatorname{IgD}$ staining were employed to study paraffin-embedded tissue blocks collected from five patients with newly diagnosed NLPHL that did not relapse or transform (single samples, cohort 1), 16 NLPHL cases with paired samples at diagnosis and the time of relapse (cohort 2), ten paired samples taken at the diagnosis of NLPHL and at the time of relapse as transformation (cohort 3 ) and 28 single sample control cases (10 with non-specific lymphadenitis, 4 with progressive transformation of germinal centers, 10 with DLBCL of an activated $B$-cell subtype, and 4 with $\mathrm{T}$-cell/histiocyte-rich large B-cell lymphoma).

Initial samples from cases that later transformed were IgD-negative, did not have the most characteristic LP cell IGHV3/IGHD3/IGHJ6 gene rearrangement, had high repertoire clonality and patients were older, as compared with cases that did not transform. Phylogenetic trees within the malignant clone in initial and relapsed and transformed samples were established. In cases that relapsed but did not transform, the LP IGH gene rearrangement was identical in initial and relapsed samples. Cases that transformed showed more complex trajectories with strong intraclonal diversification. Clonal evolution of the founder clone was seen in some transformed samples while in others, a clone from a different cell of origin was identified. The data indicate high B-cell repertoire clonality with dominant intraclonal LP cell diversification in initial NLPHL diagnostic biopsies to be a predictor of subsequent transformation and suggest a significant role of antigen drive in this process.

Another study of recombinantly-expressed B-cell receptors from microdissected LP cells from IgD-positive cases suggested that bacterial antigens from Moraxella species bind to the B-cell receptor. ${ }^{9}$ Of interest, almost one-third of the IgD-negative cases in the study by Paschold et al. shared the characteristic IGHV/D/J rearrangement of IgD-positive cases, possibly suggesting a similar pathogenesis. Moraxella catarrhalis is associated with several common childhood infectious illnesses and could be an antigenic driver. ${ }^{10}$

Paschold et al. have presented an interesting study that is a contribution to understanding the biology and pathogenesis of NLPHL and its transformation. Next-generation sequencing is performed on whole biopsy samples and avoids the difficulties of microdissection. The concept that antigens of infectious origin may be important in driving the pathogenesis, as suspected by Jackson and Parker 77 years ago, is very intriguing.

\section{Dislcosures}

No conflicts of interest to disclose.

\section{References}

1. Rappaport H, Berard CW, Butler JJ, Dorfman RF, Lukes RJ, Thoma LB. Report of the committee on histopathological criteria contributing to staging of Hodgkin's disease. Cancer Res. 1971;31(11):1864-1865.

2. Jackson H, Parker F. Hodgkin's disease. N Engl J Med. 1944;230:1-8.

3. Borchmann S, Joffe E, Moskowitz CH, et al. Active surveillance for nodular lymphocyte-predominant Hodgkin lymphoma. Blood. 2019;133(20):2121-2129.

4. Teras LR, DeSantis CE, Cerhan JR, Morton LM, Jemal A, Flower CR. 2016 US lymphoid malignancy statistics by World Health Organization subtypes. CA Cancer J Clin. 2016;66(6):443-459.

5. Al-Mansour M, Connors JM, Gascoyne RD, Skinnider B, Savage KJ. Transformation to aggressive lymphoma in nodular lymphocyte-predominant Hodgkin's lymphoma. J Clin Oncol. 2010;28(5):793-799.

6. Marafioti T, Hummel M, Anagnostopoulos I, et al. Origin of nodular lymphocyte-predominant Hodgkin's disease from a clonal expansion of highly mutated germinal-center B cells. N Engl J Med. 1997;337(7):453458.

7. Gloghini A, Bosco A, Ponzoni M, Spina M, Carbone A. Immunoarchitectural patterns in nodular lymphocyte predominant Hodgkin lymphoma: pathologic and clinical implications. Expert Rev Hematol. 2015;8(2):217-223.

8. Paschold L, Willscher E, Bein J, et al. Evolutionary clonal trajectories in nodular lymphocyte-predominant Hodgkin lymphoma with high risk of transformation. Haematologica. 2021;106(10):2654-2666

9. Thurner L, Hartmann S, Fadle N, et al. Lymphocyte predominant cells detect Moraxella catarrhalis-derived antigens in nodular lymphocytepredominant Hodgkin lymphoma. Nat Commun. 2020;11(1):2465.

10. Poppema S. Lymphocyte predominant Hodgkin lymphoma, antigendriven after alle J Pathol. 2021;253(1):1-10. 